\title{
Planar Bragg Grating Sensors Functionalized with Cyclodextrins for Trichlorofluoromethane Sensing
}

\author{
Stefan Belle ${ }^{1}$, Stefan Kefer ${ }^{1}$, Siegfried R. Waldvoge ${ }^{2}$ and Ralf Hellmann ${ }^{1}$ \\ ${ }^{1}$ Applied Laser and Photonics Group, Aschaffenburg University of Applied Sciences, Wuerzburger \\ Strasse 45, 63743 Aschaffenburg, Germany \\ 2Institiute of Organic Chemistry, Johannes Gutenberg University Mainz, Duesbergweg 10-14, 55125 \\ Mainz, Germany \\ Corresponding author's e-mail address: ralf.hellmann@th-ab.de
}

\section{Summary:}

This contribution summarizes on recent findings of planar Bragg grating sensors, functionalized with cyclodextrins, for the detection of ozone depleting trichlorofluoromethane. Detection limits as low as $5 \mathrm{ppm}$ are feasible whereas sensitivity and dynamic depend on the employed cyclodextrin class. The prospect of transferring the technology from silicas to polymer-based devices is also presented.

Keywords: Optical Sensor, Bragg Grating, Evanescent Field, Cyclodextrin, Trichlorofluoromethane

\section{Introduction}

Once an auspicious chlorofluorocarbon, widely employed as propellant and refrigerant, the usage and fabrication of trichlorofluoromethane, also referred to as CFC-11 or R-11, is now strictly prohibited due to its vast ozone depletion potential [1]. Nevertheless, the substance is still released nowadays, for example during the recycling of obsolete cooling devices or even in illegal production plants [2].

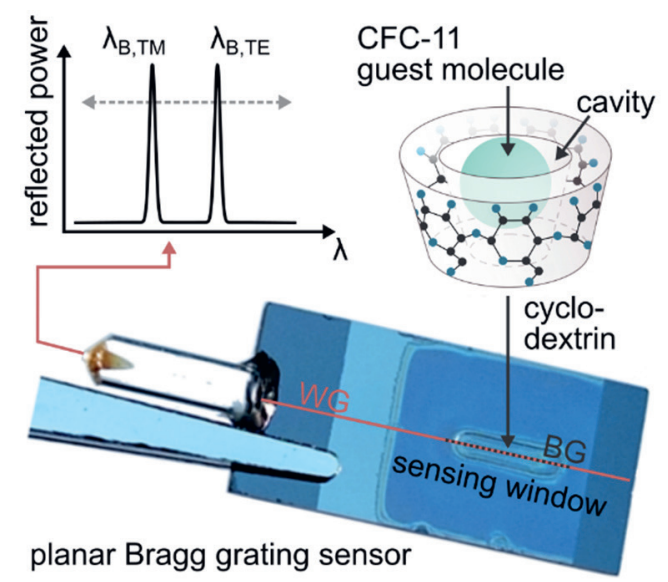

Fig. 1. Working principle of a planar Bragg grating sensor functionalized for CFC-11 sensing via cyclodextrin coatings.

Thus, reliable and sensitive detection of CFC11 is still of vital importance for the society and yet continuously a technological challenge for modern sensors. Optical Bragg gratings constitute a promising technology for this task, since, besides low weight, they offer outstanding electromagnetic, chemical and thermal resistance.
However, they necessitate functionalization for the detection of CFC-11, which can be achieved by coating the sensitive Bragg grating region with cyclodextrins (CDs). Due to their molecular structure and composition, CDs are able to form a non-covalent host-guest complex with CFC-11, as illustrated in Fig. 1. Coating a planar Bragg grating (PBG) device with CDs enables quantification of the CFC-11 molecule abundance via the evanescent field interaction of guided mode and functional coating, which leads to a shift of the PBG's modal Bragg reflection peaks $\lambda_{B, T E}$ and $\lambda_{B, T M}$. Based on their composition, CDs are classified as $\alpha$-, $\beta$ - or $\gamma$ cyclodextrin. Further modification of its solubility, viscosity and selectivity, is adapted by substitution of the CD's hydroxyl groups.

\section{Sensor Response}

An overview of the employed $C D$ derivatives is given in Tab. 1 , while Fig. 2 depicts the respective Bragg wavelength shift $\Delta \lambda_{B}$ of both modal reflection peaks as a function of the CFC-11 content, diluted in nitrogen. It is found that, in all cases, the maximum response of the TE reflection peak is about ten times larger than that of the TM signal. Per-methyl substituted derivatives exhibit maximum wavelength shifts up to

Tab. 1: Per-substituted cyclodextrin derivatives.

\begin{tabular}{|c|c|c|c|}
\hline Substitute & $\alpha-C D$ & $\beta-C D$ & $\gamma-C D$ \\
\hline Methyl & CD1 & CD2 & CD3 \\
\hline Ethyl & CD4 & CD5 & CD6 \\
\hline Allyl & CD7 & CD8 & CD9 \\
\hline
\end{tabular}




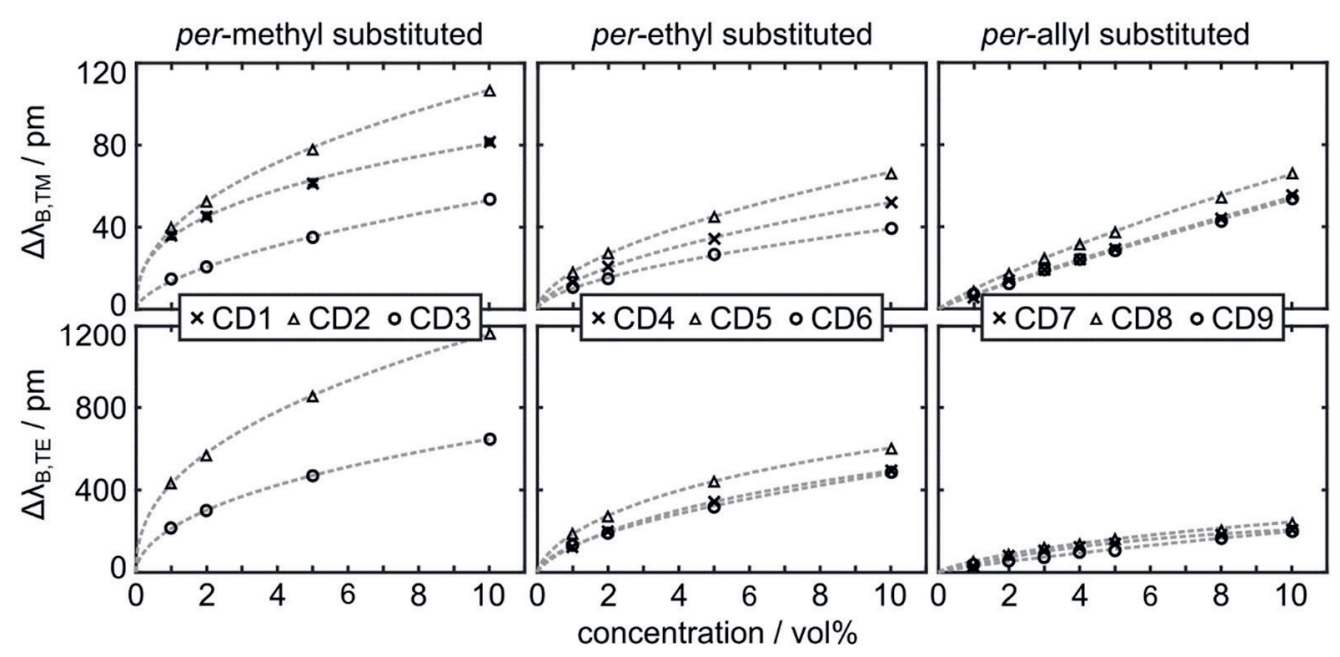

Fig. 2. Bragg wavelength shift of both modal (TE \& TM) reflection peaks as a function of CFC-11 concentration in nitrogen, for $\alpha$-, $\beta$ - or $\gamma$-cyclodextrin derivative coatings with various hydroxy substitutions.

$1200 \mathrm{pm}$ (CD2), which results in a detection limit of $5 \mathrm{ppm}$. This value is about 400 times larger than that of an uncoated PBG. The signal deflection of per-ethyl- and per-allyl substituted derivatives, however, is reduced. Albeit, in contrast to the determined sensitivities, these derivatives show a significantly faster temporal response when the sensor is exposed to CFC11 in nitrogen. For example, the rise times for CD2, CD5 and CD8, at 1 vol\% CFC-11, are $1435 \mathrm{~s}, 71 \mathrm{~s}$ and $45 \mathrm{~s}$, respectively. Consequently, it is feasible to tailor the functionalized PBG's behavior by employing the appropriate CD coating [3].

\section{Spontaneous Crystallization}

Exposing a PBG coated with CD1 to a CFC-11 content of at least $35 \mathrm{vol} \%$ leads to spontaneous crystallization of CFC-11 on the surface of the PPBG, as depicted in Fig. 3.

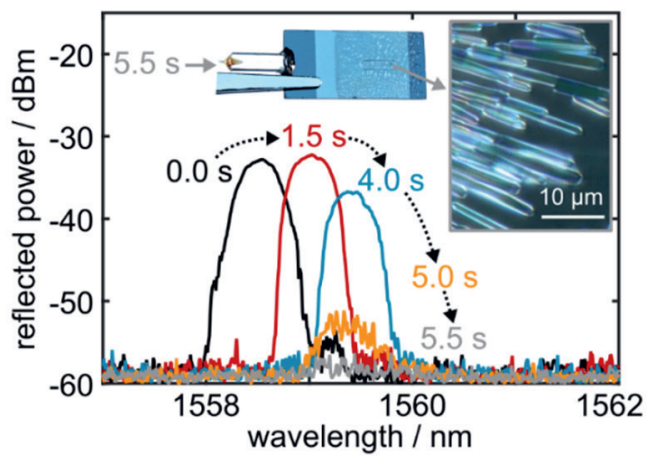

Fig. 3. Signal loss due to spontaneous crystallization. Inset: Sensor with crystallized surface and microscopic image thereof.

Within a timeframe of $5.5 \mathrm{~s}$, this leads to complete signal loss due to a drastic refractive index increase of the functionalization coating and / or scattering losses by the structural reconfiguration of the crystallized surface [4].

\section{Conclusion and Outlook}

In conclusion, PBGs functionalized with CDs are well-suited for the detection of volatile trichlorofluoromethane, whereas sensor sensitivity and response time can be adapted by employing appropriate CD derivatives. CFC-11 quantities above $35 \mathrm{vol} \%$ lead to spontaneous crystallization of the coating which can be exploited for the development of new filter and storage concepts [5]. While all PBGs used in this study are $\mathrm{SiO}_{2}$ based, future research will focus on transferring the demonstrated methodology on polymer-based planar devices and the development of new affinity materials.

\section{Acknowledgements}

We thank M. Girschikofsky (today with Heraeus Nexensos) and S. Hessler (alp group Aschaffenburg) for their valuable contributions to this research.

\section{References}

[1] M. Prather et al., The ozone layer: the road not taken, Nature 381, 551-554 (1996); doi: 10.1038/381551a0.

[2] I. Papst, Global Banks of Ozone Depleting Substances: A Country-Level Estimate, Deutsche Gesellschaft für Internationale Zusammenarbeit (GIZ) GmbH, 2017.

[3] M. Girschikofsky et al., Optical Sensor for RealTime Detection of Trichlorofluoromethane, Sensors 19 (2019); doi: 10.3390/s19030632.

[4] D. Ryvlin et al., Methyl-Substituted a-Cyclodextrin as Affinity Material for Storage, Separation, and Detection of Trichlorofluoromethane, Global Challenges 2, 1800057 (2018); doi: 10.1002/gch2.201800057.

[5] M. Girschikofsky et al., "Planar optical sensor for trichlorofluoromethane," in SPIE Future Sensing Technologies (SPIE, 2019 - 2019), 13; doi: 10.1117/12.2542013. 